\title{
Guaranteed estimation of electrochemical parameters by set inversion using interval analysis
}

\author{
I. Braems ${ }^{\text {a }}$, F. Berthier ${ }^{b, *}$, L. Jaulin ${ }^{\mathrm{a}, 1}$, M. Kieffer ${ }^{\text {a }}$, E. Walter ${ }^{\text {a }}$ \\ a Laboratoire des Signaux et Systèmes, UMR 8506, CNRS-Supélec-Université de Paris XI, 91192 Gif-sur-Yvette, France \\ ${ }^{\mathrm{b}}$ Laboratoire d'Etude des Matériaux Hors-Equilibre, UMR 8647, CNRS-Université de Paris-Sud, LEMHE, Batiment 415, 91405 Orsay, France
}

Received 15 February 2000; received in revised form 31 July 2000; accepted 31 July 2000

\begin{abstract}
The bounded-error approach to parameter estimation, mainly developed in the context of control and signal processing, is applied in the electrochemistry field in order to obtain reliable estimates for kinetic parameters. The method is based on the assumption that an uncertainty bar is available for each measurement. A set guaranteed to contain all values of the parameter vector that lead to model outputs consistent with these error bars is then computed, based on interval analysis and set inversion. The resulting technique is applied on simulated and experimental data for several classical electrochemical models. Its merits are compared to those of a more traditional approach based on least square estimation by iterative local optimization. A first obvious difference is that the point estimate provided by the latter method may or may not belong to the set estimated by the former, because it does not take the bounds on the uncertainty into account. Moreover, as our approach is global, it escapes the difficult problem of initialization encountered with iterative optimization methods. Finally, the set obtained is a natural characterization of the uncertainty on the estimated parameters. (C) 2000 Elsevier Science B.V. All rights reserved.
\end{abstract}

Keywords: Electrochemical parameters; Set inversion; Interval analysis

\section{Introduction}

The electrochemical characterization of metals involves estimating kinetic parameters from experimentally measured current as a function of potential, the simplest case being when only steady-state conditions are considered. Assume that a vector $\boldsymbol{y}$ of experimental data has been collected, and that a mathematical model depending on a vector $\boldsymbol{p}$ of unknown parameters is being built, which produces a vector $\boldsymbol{y}_{\mathrm{m}}(\boldsymbol{p})$ of model outputs. Estimating $\boldsymbol{p}$ then means tuning it to make $\boldsymbol{y}_{\mathrm{m}}(\boldsymbol{p})$ resemble $\boldsymbol{y}$, in a sense to be specified. Usually $\boldsymbol{p}$ is estimated by optimizing a criterion $j(\boldsymbol{p})$ chosen by the experimenter.

Classical estimators used in electrochemistry [1-5] proceed by minimizing a least-square criterion, i.e. they search for $\boldsymbol{p}$ that minimizes the (possibly weighted)

\footnotetext{
* Corresponding author. Fax: + 33-1-69154819.

E-mail address: fabienne.berthier@lehme.u-psud.fr (F. Berthier).

${ }^{1}$ On leave from Laboratoire d'Ingénierie des Systèmes Automatisés, Université d'Angers, France.
}

Euclidean distance between $\boldsymbol{y}$ and $\boldsymbol{y}_{\mathrm{m}}(\boldsymbol{p})$. Ideally, the resulting point estimate $\hat{\boldsymbol{p}}$ should then be a global minimizer of the cost function $j($.$) , and j(\hat{\boldsymbol{p}})$ the global minimum of the cost function. Note that for nonglobally identifiable models, several global minimizers correspond to the global minimum, thus a prior identifiability analysis [6,7] on the model may prove that there are several solutions for $\hat{\boldsymbol{p}}$. Even when the model is globally identifiable, in many situations, it turns out that the value obtained for $\hat{\boldsymbol{p}}$ depends heavily on the initial value given to the parameter vector as a result of using a local iterative method such as the Gauss-Newton, quasi-Newton or conjugate gradients algorithms. Moreover, these iterative algorithms based on local properties of the cost function may stop before reaching the expected local solution for ill-conditioned problems. The result may thus even not be a good approximation of a local minimizer.

On the other hand, global optimization methods based on a random exploration of parametric space [8], such as simulated annealing [9], genetic algorithms [10] or adaptive random search [11], have the ambition to 
bypass initialization problems. However, there is no guarantee that these methods will find good approximations of the global minimum and an associated global minimizer after a finite number of iterations.

An excellent review paper [12] describes these problems, but leaves them unsolved. In order to obtain a guaranteed characterization of the global minimizers of the cost function, one must resort to deterministic global optimization (see Refs. [13-15] for an application to a problem of maximum-likelihood parameter estimation similar to those considered here). Guaranteed as they may be, these methods are once again point estimators that merely estimate one or several numerical values for $\hat{\boldsymbol{p}}$.

The purpose of this paper is to advocate a promising alternative approach, so far developed in the fields of control and signal processing. This approach is known under the name of bounded-error estimation, or set membership estimation, (see e.g. Refs. [16-19] and references therein), and the version implemented here is global and guaranteed. To the best of our knowledge, it has never been used in electrochemistry. This approach encompasses all the acceptable values of the parameter vector in a set that is characterized. The extent of this set informs us about the uncertainty with which the parameters are estimated [20,21]. The methodology is illustrated by estimating kinetic constants in electrochemical reaction mechanisms presented in Section 2. The notion of set inversion and the algorithm to be used are introduced in Section 3. Section 4 describes the results obtained from steady-state data on the mechanisms of Section 2 and illustrates the advantages of such a guaranteed set estimation.

\section{Reaction mechanisms}

\subsection{Redox model}

Consider the classical redox system described as:

$\mathrm{O}+\mathrm{e}^{-} \leftrightarrow \mathrm{R}$

Under steady state conditions, the evolution of the Faradaic current density as a function of the dimensionless electrode voltage $\xi$ is given by [18]:

$j_{\mathrm{f}}=F k^{\circ} \frac{c_{\mathrm{R}, \text { init }} \exp \left(\alpha_{\mathrm{o}} \xi\right)-c_{\mathrm{O}, \text { init }} \exp \left(-\alpha_{\mathrm{r}} \xi\right)}{1+k^{\circ}\left(\frac{\exp \left(\alpha_{\mathrm{o}} \xi\right)}{m_{\mathrm{R}}}+\frac{\exp \left(-\alpha_{\mathrm{r}} \xi\right)}{m_{\mathrm{O}}}\right)}$

with $k^{\circ}=k_{\mathrm{o}}^{\alpha_{\mathrm{o}}} k_{\mathrm{r}}^{\alpha_{\mathrm{r}}}$ and $\xi=f\left(E-E^{\circ}\right)$

In these equations $k^{\circ}$ is the kinetic constant of the standard electronic transfer and $E^{\circ}$ the standard potential; $\alpha_{\mathrm{o}}$ and $\alpha_{\mathrm{r}}$ are symmetry factors for the reaction, $k_{\mathrm{o}}$ and $k_{\mathrm{r}}$ are the associated kinetic constants. $m_{\mathrm{O}}$ and $m_{\mathrm{R}}$ are mass transfer constants. $F$ is the Faraday constant,
$f=F / R T$, where $T$ is the absolute temperature and $R$ the ideal gas constant, $c_{\mathrm{O} \text {,init }}$ and $c_{\mathrm{R} \text {,init }}$ are the initial concentrations of $\mathrm{O}$ and $\mathrm{R}$.

Assuming that $m_{\mathrm{O}}, m_{\mathrm{R}}, E^{\circ}$ are known, we define the parameter vector as:

$\boldsymbol{p}=\left(p_{1} p_{2}\right)^{\prime}=\left(\frac{1}{k^{\circ}} \alpha_{\mathrm{O}}\right)^{\prime}$

where $\boldsymbol{X}^{\prime}$ is the transposed vector of the vector $\boldsymbol{X}$, and the system output as:

$y^{1}(t)=\frac{F}{j_{\mathrm{f}}}\left(c_{\mathrm{R}, \text { init }}-c_{\mathrm{O}, \text { init }} \exp (-t)\right)-\frac{1}{m_{\mathrm{R}}}-\frac{\exp (-t)}{m_{\mathrm{O}}}$

with

$t=\xi$

Using Eq. (2), the model of $y^{1}$ can be then written as:

$y_{\mathrm{m}}^{1}(\boldsymbol{p}, t)=p_{1} \exp \left(-p_{2} t\right)$

which is obviously globally identifiable. Note that Eq. (7) is a particular case of the sum of exponentials model also used in the following paragraph.

\subsection{Volmer-Heyrovsky model}

The Volmer-Heyrovsky mechanism is proposed to describe the dissolution-passivation of metals, as follows:

$\mathrm{M}, \mathrm{s}+\mathrm{A}^{-} \stackrel{k_{1}}{\rightarrow} \mathrm{MA}, \mathrm{s}+\mathrm{e}^{-}$

$\mathrm{MA}, \mathrm{s} \stackrel{k_{2}}{\rightarrow} \mathrm{MA}^{+}+\mathrm{s}+\mathrm{e}^{-}$

The first step describes the oxidation of a metal M,s by electrosorption of some species $\mathrm{A}^{-}$as MA,s. The second step (electrodesorption) regenerates a superficial free site. Each one of these steps produces one electron. Assuming that the interfacial depletion in $\mathrm{A}^{-}$is negligible (so the interfacial concentration of $\mathrm{A}^{-}$is equal to $\left.c_{\mathrm{A} \text {,init }}\right)$, and that the two steps are irreversible, under steady-state conditions, the kinetics of an electrode reaction following this mechanism are then described by:

$j_{\mathrm{f}}(E)=2 F \Gamma \frac{c_{\mathrm{A}, \text { init }} k_{\mathrm{O} 1} \exp \left(\alpha_{\mathrm{O} 1} f E\right) k_{\mathrm{O} 2} \exp \left(\alpha_{\mathrm{O} 2} f E\right)}{c_{\mathrm{A}, \text { init }} k_{\mathrm{O} 1} \exp \left(\alpha_{\mathrm{O} 1} f E\right)+k_{\mathrm{O} 2} \exp \left(\alpha_{\mathrm{O} 2} f E\right)}$

Taking $t=f E$ and defining the system output by:

$y^{2}(t)=\frac{2 F \Gamma}{j_{\mathrm{f}}(E)}$

the corresponding model output can then be written as:

$$
\begin{aligned}
& y_{\mathrm{m}}^{2}(\boldsymbol{p}, t)=\frac{1}{k_{\mathrm{O} 1} \exp \left(\alpha_{\mathrm{O} 1} t\right)}+\frac{c_{\mathrm{A}, \text { init }}}{k_{\mathrm{O} 2} \exp \left(\alpha_{\mathrm{O} 2} t\right)} \\
& =p_{1} \exp \left(-p_{3} t\right)+p_{2} \exp \left(-p_{4} t\right)
\end{aligned}
$$


where:

$\boldsymbol{p}=\left(\frac{1}{k_{\mathrm{O} 1}} \frac{c_{\mathrm{A}, \text { init }}}{k_{\mathrm{O} 2}} \alpha_{\mathrm{O} 1} \alpha_{\mathrm{O} 2}\right)^{\prime}$

From Eq. (12) one readily sees that interchanging the roles of $\left(p_{1}, p_{3}\right)$ and $\left(p_{2}, p_{4}\right)$ does not modify $\boldsymbol{y}_{\mathrm{m}}^{2}$ Thus the vectors:

$$
\begin{gathered}
\boldsymbol{p}^{a}=\left(\frac{1}{k_{\mathrm{O} 1}} \frac{c_{\mathrm{A}, \text { init }}}{k_{\mathrm{O} 2}} \alpha_{\mathrm{O} 1} \alpha_{\mathrm{O} 2}\right)^{\prime} \text { and } \\
\boldsymbol{p}^{b}=\left(\frac{c_{\mathrm{A}, \text { init }}}{k_{\mathrm{O} 2}} \frac{1}{k_{\mathrm{O} 1}} \alpha_{\mathrm{O} 2} \alpha_{\mathrm{O} 1}\right)^{\prime}
\end{gathered}
$$

are such that $\boldsymbol{y}_{\mathrm{m}}\left(\boldsymbol{p}^{a}, t\right)=\boldsymbol{y}_{\mathrm{m}}\left(\boldsymbol{p}^{b}, t\right)$ for all $t$. The parameter vector $\boldsymbol{p}$ is therefore not uniquely identifiable from such a steady state experiment. See [6,7,22,23] for more details.

We will now introduce the framework to be used to obtain guaranteed estimates of the parameters of these models.

\section{Set inversion using interval analysis}

In this section, we briefly recall the main ideas of bounded-error estimation viewed as a problem of set inversion, and present tools based on interval analysis that can be applied in the nonlinear context of the electrochemical models.

Even when all the global minimizers of a suitably defined cost function can be found, they form a very incomplete answer to the parameter estimation problem, because values of the parameter vector that do not correspond to global minimizers may still be perfectly acceptable given the uncertainty in the experimental data. This motivates the interest for a family of techniques known under the generic name of set membership estimation or bounded-error estimation (see e.g. Refs. [16-19] and references therein).

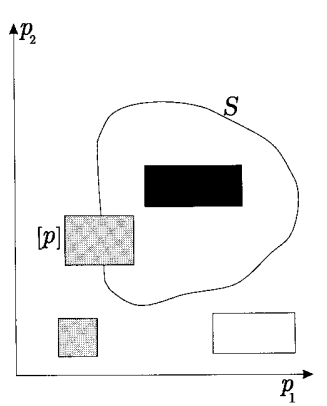

parameter space

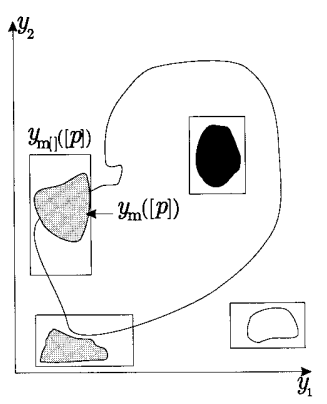

data space
Fig. 1. Boxes and their images by $\boldsymbol{y}_{\mathrm{m}}$ and $\boldsymbol{y}_{\mathrm{m}}$ :unfeasible (white), undetermined (light grey), feasible (black).

\subsection{Bounded-error estimation}

For various reasons (impossibility to repeat experiments, small number of data points, lack of time...) the statistical distribution of the noise associated with experimental data is often inaccessible. On the other hand, sensors are characterized in their technical data sheets by their maximum absolute measurement errors on any given range of operation, and this information can be used to define the largest discrepancy between the model output and the corresponding measurement one is prepared to accept. We call error the difference between the experimental value $\boldsymbol{y}$ and the theoretical value $\boldsymbol{y}_{\mathrm{m}}$ as computed by the model and uncertainty the upper bound of the absolute value of this error. From the vector $\boldsymbol{y}$ of all available experimental data and their uncertainty the set $[\boldsymbol{y}]$ of all acceptable values for the vector $\boldsymbol{y}_{\mathrm{m}}$ can be built. An estimate $\hat{\boldsymbol{p}}$ of the parameter vector will be deemed acceptable if and only if $\boldsymbol{y}_{\mathrm{m}}(\hat{\boldsymbol{p}}) \in[\boldsymbol{y}]$. Thus, bounded-error estimation consists in determining the set $S$ of all $\boldsymbol{p}$ 's in some prior domain of interest $\boldsymbol{P}$ that are such that $\boldsymbol{y}_{\mathrm{m}}(\boldsymbol{p})$ is consistent with the data and their uncertainty:

$S=\left\{\boldsymbol{p} \in \boldsymbol{P} \mid \boldsymbol{y}_{\mathrm{m}}(\boldsymbol{p}) \in[\boldsymbol{y}]\right\}$

In this paper, uncertainty was quantified from the information contained in the technical data for the electrochemical interface [24].

Bounded-error estimation presents the following advantages compared to the usual statistical approaches:

1. Structural model errors are readily accommodated (deterministic).

2. The tools of interval analysis turn out to be more efficient for this type of problem than for problems of global minimization of a cost function.

3. Contrary to the minimization of the cost function, it guarantees that the error between the model output and the corresponding data point remains acceptable at all data points (see Fig. 1).

\subsection{Set inversion}

Eq. (14) can be rewritten as:

$S=\boldsymbol{y}_{\mathrm{m}}^{-1}([\boldsymbol{y}]) \cap \boldsymbol{P}$

Thus, characterizing $S$ is a set-inversion problem, since we have to compute the reciprocal image of a set $\left[\boldsymbol{y}\right.$ ] by the function $\boldsymbol{y}_{\mathrm{m}}$. The computation of a guaranteed approximation of $S$ should be based on interval analysis, which provides effective tools to define relations, operators and functions on intervals and boxes.

\subsection{Interval analysis}

To real intervals (i.e. finite, closed and connected subsets of $\mathbb{R}$ ), an arithmetic can be associated, based on 
operations and relations. Object-oriented languages such as $\mathrm{C}++$ allow one to manipulate intervals on computers easily and in a guaranteed way. We present here only the few basic notions and notation needed to understand this paper, for additional information, see e.g. Refs. [25-27].

We note a scalar interval $[p]$ of $\mathbb{R}$ as:

$[p]=\left[p^{-} ; p^{+}\right]=\left\{p \mid p^{-} \leq p \leq p^{+}\right\}$

The four basic arithmetic rules on intervals are simply derived from the classic ones for addition, subtraction, multiplication and division.

A box (or vector interval) $[\boldsymbol{p}]$ of $\mathbb{R}^{n_{\mathrm{P}}}$ is the Cartesian product of $n_{\mathrm{p}}$ scalar intervals:

$[\boldsymbol{p}]=\left[\boldsymbol{p}^{-} ; \boldsymbol{p}^{+}\right]=\left[p_{1}^{-} ; p_{1}^{+}\right] \times \ldots \times\left[p_{1}^{-} ; p_{1}^{+}\right] \times \ldots \times\left[p_{\mathrm{np}}^{-} ; p_{\mathrm{np}}^{+}\right]$

The width $w([\boldsymbol{p}])$ of a box $[\boldsymbol{p}]$ is the length of its largest interval component. The interval extension of a function $f$ is denoted by $[f]$ and defined as:

$[f]:[p] \rightarrow[f(p) \mid p \in[p]]$

where $[X]$ is the smallest box including the set $X$. $[f]([p])$ is often impossible to evaluate. We then approximate $[f]$ by a so-called inclusion function, denoted by $\boldsymbol{f}_{[]}$which can be evaluated. Let $f$ be a continuous function from $\mathbb{R}^{n_{P}}$ to $\mathbb{R}^{N}$. $\boldsymbol{f}_{[]}$is an inclusion function for $\boldsymbol{f}$ if for any box $[\boldsymbol{p}]$ of $\mathbb{R}^{n_{P}}, \boldsymbol{f}_{[]}([\boldsymbol{p}])$ is a box of $\mathbb{R}^{N}$ satisfying:

$f([p]) \subset f_{[]}([p])$

$w([\boldsymbol{p}]) \rightarrow 0 \Rightarrow w\left(\boldsymbol{f}_{[]}([\boldsymbol{p}])\right) \rightarrow 0$

\subsubsection{Example 1:}

Consider the function:

$\mathrm{f}: \mathbb{D} \rightarrow \mathbb{R}$

$x \rightarrow x^{2}-2 x$

with $\mathbb{D}=[0 ; 1]$. A first inclusion function is obtained by replacing all computations on real numbers by computations on their interval counterparts. Thus:

$f_{1}([0 ; 1])=[0 ; 1]^{2}-2[0 ; 1]=[0 ; 1][0 ; 1]-2[0 ; 1]$

$f_{1}([0 ; 1])=[0 ; 1]-[0 ; 2]=[-2 ; 1]$

Another possibility is to rewrite $f(x)$ as $(x-1)^{2}-1$, which leads to:

$$
\begin{aligned}
f_{2}([0 ; 1]) & =([0 ; 1]-1)^{2}-1=[-1 ; 0]^{2}-1=[0 ; 1]-1 \\
& =[-1 ; 0]
\end{aligned}
$$

$f_{2}([0 ; 1])$ is a more accurate enclosure of $f([0 ; 1])$ than $f_{1}([0 ; 1])$. This is due to the fact that $[x]$ appears twice in $f_{1}$ but once in $f_{2}$. It is always a good practice to write $f$ in such a way as to decrease the number of occurrences of each variable.

\subsubsection{Example 2:}

Consider the function:

$f: \mathbb{R}^{2} \rightarrow \mathbb{R}$

$\boldsymbol{P} \rightarrow p_{1} \exp \left(-p_{2} t\right)$

with $p_{1} \in\left[p_{1}\right], p_{2} \in\left[p_{2}\right]$, which corresponds to Eq. (7). A natural inclusion function is:

$\mathrm{f}_{[}([\mathbf{P}])=\left[p_{1}\right] \exp \left(-\left[p_{2}\right] t\right)$

The number of occurrences of each of the two variables is one, so this inclusion function will be as accurate as possible.

Note that some free downloadable software already exists to perform interval computations (see Refs. $[28,29])$.

\subsection{SIVIA}

The SIVIA algorithm (an acronym for Set Inversion via Interval Analysis) solves set-inversion problems in an effective and guaranteed way. It is recursive and explores $P$ systematically and exhaustively (contrary to random-search methods), by means of bisections. The main idea is to partition $P$ into three unions of boxes, or subpavings:

- the feasible boxes that have been proved to belong to $S$,

- the unfeasible boxes that have been proved to have an empty intersection with $S$,

- the undetermined boxes for which nothing has been proved so far.

Whenever possible, interval analysis will be used to prove that a box is feasible or unfeasible, by taking advantage of the following implications:

$\boldsymbol{y}_{m[}([\boldsymbol{P}]) \subset[\boldsymbol{y}] \Rightarrow[\boldsymbol{P}] \subset S$

$\boldsymbol{y}_{\mathrm{m}[]}([p]) \cap[y]=\varnothing \Rightarrow[p] \cap S=\varnothing$

\subsubsection{Example 3:}

Since $\boldsymbol{y}_{\mathrm{m}[\mathrm{l}}([\boldsymbol{p}])$, which has been obtained by interval calculus, is a box, the two tests $\boldsymbol{y}_{\mathrm{m}[]}([\boldsymbol{p}]) \subset[\boldsymbol{y}]$ and $\boldsymbol{y}_{\mathrm{m}[\mathrm{J}}([\boldsymbol{p}]) \cap[\boldsymbol{y}]=\varnothing$ are easily evaluated.

Assume that $y_{m}(\boldsymbol{p})=p_{1}^{2}+p_{2}^{2},[y]=[1 ; 2]$, and $P=[-2 ; 2] \times[-2 ; 2]$. The set $S$ is then defined by:

$S=\left\{\left(p_{1}, p_{2}\right) \in P \mid y_{m}\left(p_{1}, p_{2}\right)=p_{1}^{2}+p_{2}^{2} \in[1: 2]\right\}$

For $[\mathrm{p}]=[-0.1 ; 0.1] \times[-0.1 ; 0.1]$

$y_{m[]}([\mathbf{P}])=y_{m[]}([-0.1 ; 0.1],[-0.1 ; 0.1])=[0 ; 0.02]$

which has an empty intersection with [y]. From Eq. (20) we know that $[\mathbf{P}] \cap S=\varnothing$. Now for $[\boldsymbol{p}]=[0.9 ; 1] \times$ $[0.8 ; 1], \boldsymbol{y}_{\mathrm{m} \square}([0.9 ; 1],[0.8 ; 1])=[1.45 ; 2] \subset[y]$, so from Eq. (19) we know that $[\boldsymbol{p}] \subset S$. At last, for $[\mathbf{P}]=[-2 ; 2] \times$ [-2;2], we get 


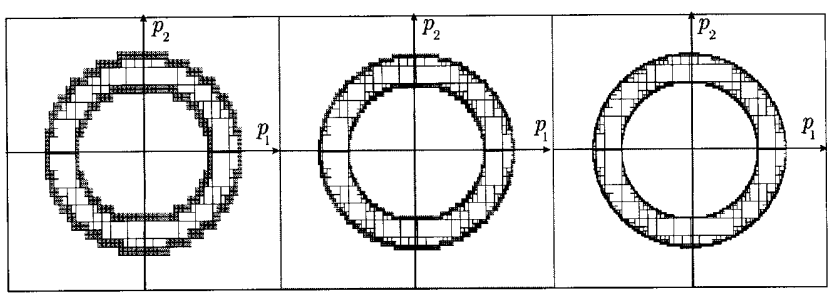

Fig. 2. Characterization of $S$ as defined in Example 2; left $\varepsilon=0.08$, center $\varepsilon=0.04$; right $\varepsilon=0.02$.

$y_{\mathrm{m}[}([\mathbf{P}])=[-2 ; 2]^{2}+[-2 ; 2]^{2}=[0 ; 8]$

Neither Eqs. (19) or (20) is satisfied and nothing has been proved yet about the feasibility of $[\mathbf{P}]$.

Whenever the tests Eqs. (19) and (20) are inconclusive, one may bisect $[\boldsymbol{p}]$ into two subboxes on which the same analysis can be carried out. To avoid iterating forever, we introduce a tolerance parameter $\varepsilon$ to be tuned by the user. Thus, unless its width is smaller than $\varepsilon$, any box $[\boldsymbol{p}]$ on which the tests Eqs. (19) and (20) are inconclusive is bisected, and each resulting subbox is then tested. Any box with width inferior or equal to $\varepsilon$ for which no conclusion can be reached is not bisected any further but kept instead in a list $S^{-}$of undetermined boxes. The resulting recursive set-inversion algorithm $\operatorname{SIVIA}([\boldsymbol{p}], \varepsilon)$ can be summarized as follows: (initially, $S^{-}=\varnothing$ and $\Delta S=P$ ).

Input $\quad[p], \varepsilon$

Step 1 if $w([\boldsymbol{p}]$ then $[\boldsymbol{p}] \leadsto \Delta S$ (list of undetermined boxes)

Step 2 if $\boldsymbol{y}_{m \square}([\boldsymbol{p}]) \subset[\boldsymbol{y}]$, then $[\boldsymbol{p}] \leadsto S^{-}$(list of feasible boxes)

Step 3 if $\boldsymbol{y}_{m[}([\boldsymbol{p}]) \cap[\boldsymbol{y}]=\varnothing$ then return

Step $4 \quad \operatorname{Bisect}\left([\boldsymbol{p}],\left[\boldsymbol{p}_{1}\right],\left[\boldsymbol{p}_{2}\right]\right)$;

$\operatorname{SIVIA}([\boldsymbol{p}], \varepsilon) ; \operatorname{SIVIA}\left(\left[\boldsymbol{p}_{2}\right], \varepsilon\right)$

Upon completion, after a finite number of steps, we have

$S^{-} \subset S \subset\left(S^{-} \cup \Delta S\right)$ which means that the unknown set $S$ has been bracketed, in the sense of inclusion, between two known sets. The distance between $S^{-}$and $S^{-} \cup \Delta S$ is an indication of the precision with which $S$ has been characterized. We will call the outer approximation of $\mathrm{S}$ the set $S^{+}=S^{-} \cup \Delta S$

Example 4 (end of Example 3). Consider again the set $S$ defined by Eq. (21): Characterizing it is a set inversion problem, since we want to estimate the reciprocal image of the interval $[1 ; 2]$ by the function $f$. The search space $P=[-2 ; 2] \times[-2 ; 2]$ is bounded by the frame of Fig. 2. The $\Delta S$ subpaving (in grey) contains the boundary of $S$, while $S^{-}$is included in $S$. From left to right, the accuracy of the description is improved by decreasing $\varepsilon$, at the cost of more computation. For instance, $\quad[\boldsymbol{p}]=[-0.01 ; 0.01] \times[-1 ;-0.99] ; \quad y_{\mathrm{m} \square}([\mathbf{P}])=$ $[0.9801 ; 1.0001]$ is undetermined and stored in $S^{-}$for $\varepsilon=0.08$ because $w([\boldsymbol{p}])=0.02$; but it is bisected for any $\varepsilon$ smaller than 0.02 .

\section{Application to electrochemical models}

We now consider two applications taken from electrochemistry. First we study the redox model, on simulated and real data. This simple two-parameter model, which is globally identifiable, will be used to illustrate the behavior of SIVIA.

The study of the Volmer-Heyrovsky model demonstrates the capacity of the approach to characterize the entire feasible region for the parameter vector, when usual point estimators fail.

\subsection{Redox model}

In order to stress the influence of uncertainty, we first study $S$ as a function of the volume of [y], on simulated data. For the true parameter vector $\boldsymbol{p}^{*}=\left(\begin{array}{lll}100.4 & { }^{\prime} \text { and }\end{array}\right.$ a noise on the current density uniformly distributed in [ $-0.01 ; 0.01]$, Fig. 3 presents the sets obtained for an uncertainty on $j_{\mathrm{f}}$ taken as: (a) 0.1 , (b) 0.01 . The search box is $P=\left[10^{-6} ; 10^{6}\right] \times[0 ; 1]$. Unlike results from other methods, a guaranteed solution set is obtained. The simple version of SIVIA presented here makes no at-
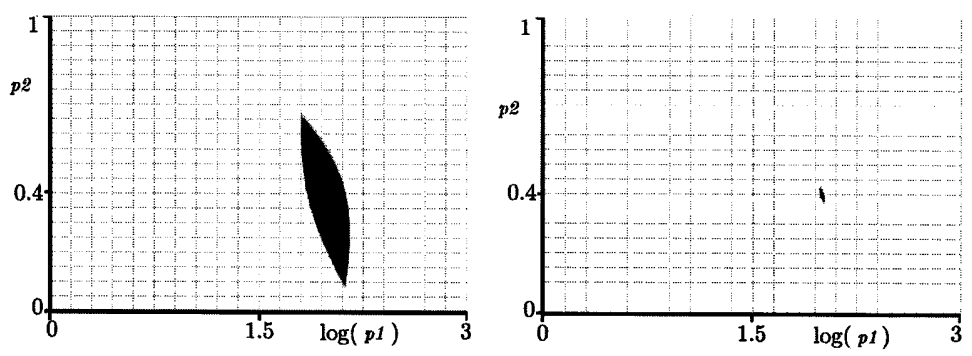

Fig. 3. Results provided by SIVIA for simulated data (redox model). Left: the uncertainty is ten times larger than on the right; parameter uncertainity estimates are obtained by projecting these sets on each line. 
Table 1

Computational time $t_{\mathrm{c}}$ and number of bisections $n$ for decreasing tolerance parameter $\varepsilon$, for the simple version of SIVIA presented here $^{\text {a }}$

\begin{tabular}{lrr}
\hline$\varepsilon$ & \multicolumn{1}{c}{$t_{\mathrm{c}}$} & \\
\hline 1 & 0.026 & 36 \\
0.5 & 0.036 & 62 \\
0.1 & 0.161 & 310 \\
0.01 & 2.170 & 3859 \\
0.001 & 19.551 & 33359 \\
0.0001 & 330.585 & 538971 \\
\hline
\end{tabular}

${ }^{\mathrm{a}}$ As the number of bisections quickly increases when one improves the accuracy, the computational time increases exponentially.

tempt at minimizing the computational time $t_{\mathrm{c}}$, which depends on the tolerance parameter $\varepsilon$. As $\varepsilon$ decreases, the number of bisections $n$ and thus $t_{\mathrm{c}}$ increase exponentially (see Table 1). Recent techniques based on constraint propagation make it possible to improve $t_{\mathrm{c}}$ by reducing $n$, with the help of any contraction algorithm [30,31]. On a $350 \mathrm{MHz}$ Pentium II, it then becomes possible to obtain a good estimate $(\varepsilon=0.001)$ in less than five seconds.

An uncertainty interval for each parameter can be obtained by projection of the outer approximation of $S$ onto the axes of parameter space: (a) for an uncertainty on $j_{\mathrm{f}}$ taken as $0.1, p_{1} \in[63.487 ; 141.457], p_{1} \in[0.085 ; 0.668]$ : (b): if this uncertainty is decreased to 0.01 , the parameter uncertainty intervals become $p_{2} \in[96.141 ; 103.908]$, $p_{2} \in[0.370 ; 0429]$.

For an uncertainty of $0.001, S$ becomes empty because SIVIA has eradicated all $\boldsymbol{p}$ 's in $P$, thus proving that there is no estimate consistent with our data and hypotheses, something no point estimator can do.

Actual data for a redox system were collected for the system $\mathrm{Fe}(\mathrm{CN})_{6}^{3-} / \mathrm{Fe}(\mathrm{CN})_{6}^{4-}$ in $\mathrm{KCl}(0.5 \mathrm{M})$, $\mathrm{Fe}(\mathrm{CN})_{6}^{3-}\left(10^{-2} \mathrm{M}\right)$, studied with a rotating electrode (2000 rpm). A Solartron Schlumberger 1286 potentiostat was employed, driven by SAMDIE acquisition software [32].

Consider the model in Eq. (7) with $\boldsymbol{p}$ defined by Eq. (4), and take:

$$
\begin{gathered}
c_{\mathrm{O}, \text { init }}=10^{-5} \mathrm{~mol} \mathrm{~cm}^{-3}, c_{\mathrm{R}, \text { init }}=0 \mathrm{~mol} \mathrm{~cm}^{-3}, \\
v=0.977 \times 10^{-2} \mathrm{~cm}^{2} \mathrm{~s}^{-1} \\
\omega=2000 \times \frac{\pi}{30} \mathrm{rad} \mathrm{s}^{-1}, \quad E^{\circ}=0.223 \mathrm{~V} / \mathrm{SHE}
\end{gathered}
$$

Classically, the estimates of the parameter vector are obtained on the Tafel lines. By fitting asymptotes one gets a particular model output which may or may not fall within the template as this template is ignored by the method (see Fig. 4). Moreover the information provided by the non-asymptotic region is then not taken into account. The set-inversion approach presented in this paper allows one to get the set of all acceptable parameter vectors $\boldsymbol{p}$ for which $\boldsymbol{y}_{\mathrm{m}}(\mathbf{p})$ is included in the template defined by $[\boldsymbol{y}]$.

The solution set $S$ obtained by SIVIA turns out to be empty, which implies that one hypothesis at least is not satisfied. Indeed, the faradaic current density is positive at high electrode potentials, so data for large $\xi$ do not belong to the template defined by $[\boldsymbol{y}]$, assuming that $c_{\mathrm{R}, \text { init }}=0$. This hypothesis should therefore be questioned.

We then define the model as:

$$
y_{\mathrm{m}}^{3}(\boldsymbol{p}, t)=\frac{p_{3}-c_{\mathrm{O}, \text { init }} \exp (-t)}{p_{1} \exp \left(-p_{2} t\right)+\frac{1}{m_{\mathrm{O}}}+\frac{1}{m_{\mathrm{R}}} \exp (-t)}
$$

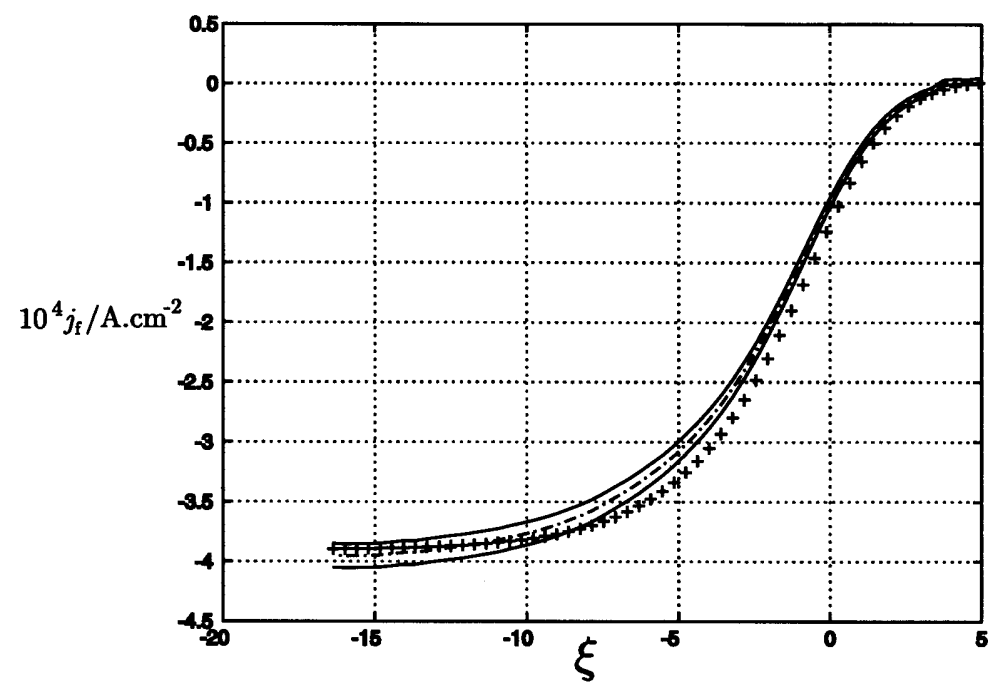

Fig. 4. Relation $j_{\mathrm{f}}(\xi)$ : experimental data (mixed line), template deduced from uncertainty in these data (full line), model output for the parameter vector obtained by the Tafel method (+). 
Table 2

Results obtained by SIVIA on the redox example, with $0 \varepsilon=0.0001$

\begin{tabular}{llll}
\hline$i$ & $p_{i}$ & $P_{i}$ & {$\left[\hat{p}_{\mathrm{i}}\right]$} \\
\hline 1 & $1 / k^{\circ}$ & {$\left[0.01 ; 10^{6}\right]$} & {$[199.522 ; 314.580]$} \\
2 & $\alpha_{\mathrm{o}}$ & {$[0 ; 1]$} & {$[0.562 ; 0.743]$} \\
3 & $c_{\mathrm{R}, \text { init }}$ & {$\left[0 ; 10^{5}\right]$} & {$\left[1.937 \times 10^{7} ; 2.361 \times 10^{7}\right]$} \\
4 & $1 / m_{\mathrm{o}}$ & {$\left[0.1 ; 10^{5}\right]$} & {$[132.517 ; 235.526]$} \\
5 & $1 / m_{\mathrm{o}}$ & {$\left[0.1 ; 10^{5}\right]$} & {$[147.340 ; 157.596]$} \\
\hline
\end{tabular}

$\boldsymbol{p}=\left(\frac{1}{k^{\circ}} \alpha_{\mathrm{O}} c_{\mathrm{R}, \text { init }}\right)^{\prime}$

The outer approximation $S^{+}$of $S$ computed by SIVIA is then non-empty and enclosed in the box

$$
\text { [263.652;299.212] } \times[0.581 ; 0.632]
$$$$
\times\left[2.039 \times 10^{-7} ; 2.191 \times 10^{-7}\right] .
$$

Moreover the list $S^{-}$is non-empty, which proves that $S$ is not empty. Notice that $c_{\mathrm{R}, \text { init }} \in\left[2.039 \times 10^{-7} ; 2.191 \times\right.$ $10^{-7}$, which rules out a zero value. Asymptotic methods do not allow the estimation of $c_{\mathrm{R} \text {,init }}$ with this accuracy based on the present data, as they do not take into account the non-asymptotic region and the upper bound for the dimensionless electrode voltage $\xi$ is too low.

To escape this limitation of asymptotic methods and take the data associated with mixed regimes into account, one may think to use nonlinear programming to estimate $\boldsymbol{p}$ by least-squares. Again, there would be no guarantee that the resulting method would be consistent with the template defined by the uncertainty of the sensors.

So far, the diffusion coefficients and the standard potential were assumed to be known. We assume now that they are unknown. An extended parameter vector can then be defined as: $\boldsymbol{p}=\left(\frac{1}{k^{\circ}} \alpha_{\mathrm{o}} c_{\mathrm{R}, \text { init }} \frac{1}{m_{\mathrm{O}}} \frac{1}{m_{\mathrm{R}}}\right)^{\prime}$

Table 2 presents the results obtained by SIVIA, after projecting $S^{+}$onto each of the parameter axes.

The values taken previously for $m_{\mathrm{O}}$ and $m_{\mathrm{R}}$ were consistent with these estimates, and the relationship $m_{\mathrm{O}}=m_{\mathrm{R}}$ that had been assumed is not invalidated as the intersection between the intervals $\left[\hat{p}_{4}\right]$ and $\left[\hat{p}_{5}\right]$ is non-empty. This assumption can however be dispensed with, since $m_{\mathrm{O}}$ and $m_{\mathrm{R}}$ can be estimated separately. Since $\hat{m}_{\mathrm{O}} \in\left[4.24 \times 10^{-3} ; 7.55 \times 10^{-3}\right]$ and $\hat{m}_{\mathrm{R}} \in[6.34 \times$ $\left.10^{-3} ; 6.79 \times 10^{-3}\right]$, it turns out that $m_{\mathrm{O}}$ is estimated more precisely than $m_{\mathrm{R}}$, which is not surprising because most of the data correspond to reduction. To improve the precision on $m_{\mathrm{R}}$, one should explore the anodic behavior.

\subsection{Volmer-Heyrovsky model}

First consider noise-free simulated data:

$$
\begin{aligned}
& y\left(t_{i}\right)=10^{-5} \exp \left(-0.4 t_{i}\right)+10^{-9} \exp \left(-0.8 t_{i}\right) ; \\
& \quad i=1, \ldots, 81,
\end{aligned}
$$

which were generated by Eq. (12) with:

$\boldsymbol{p}^{*}=\left(\begin{array}{llll}10^{-5} & 10^{-9} & 0.4 & 0.8\end{array}\right)^{\prime}$

for $c_{\mathrm{A} \text {,init }}=10^{-3} \mathrm{~mol} \mathrm{~cm}{ }^{-3}$. The independent variable $t_{\mathrm{i}}$ varies between -11.61 and 19.35 as we consider

$E \in[-0.3 \mathrm{~V} ; 0.5 \mathrm{~V}]$

We assume that:

$P=P^{1}=\left(\left[10^{-9} ; 10^{6}\right]\left[10^{-9} ; 10^{6}\right][0 ; 1][0 ; 1]\right)^{\prime}$

The projection on the plane $\left(p_{3}, p_{4}\right)$ of the set $S^{0} \cup S^{-}$ obtained by SIVIA is presented on Fig. 5, left. As could be expected, it contains two symmetrical point images

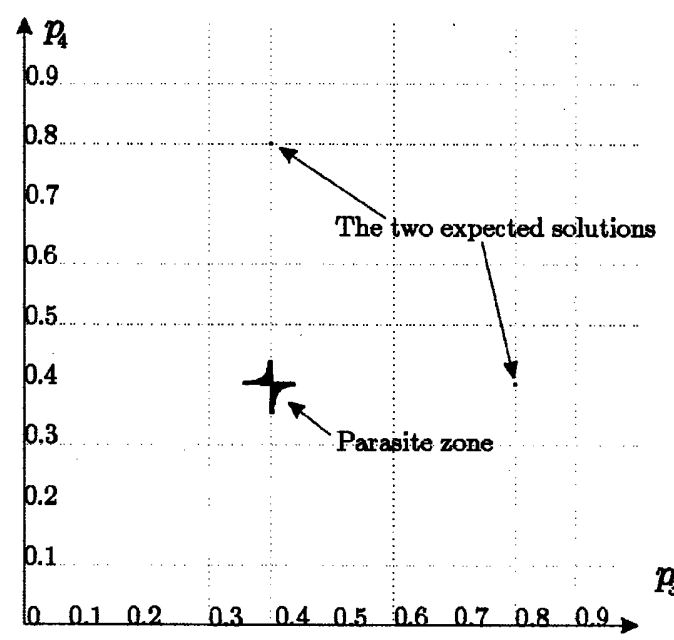

Fig. 5. Projection in the plane $(p 3, p 4)$ of the set $S^{+}$computed by SIVIA, (left: no noise, search on $P^{1}$; right: $5 \%$ noise, search on the limited region $P^{2}$, see text). 
Table 3

Results obtained by minimization of a least-square criterion by the Gauss-Newton algorithm on the Volmer-Heyrovsky example

\begin{tabular}{llc}
\hline$i$ & $p_{i}^{*}$ & $\hat{\boldsymbol{p}}_{i}$ \\
\hline 1 & $10^{-5}$ & $3.57 \times 10^{-7}$ \\
2 & $10^{-9}$ & $9.622 \times 10^{-6}$ \\
3 & 0.4 & 0.4655 \\
4 & 0.8 & 0.3978 \\
\hline
\end{tabular}

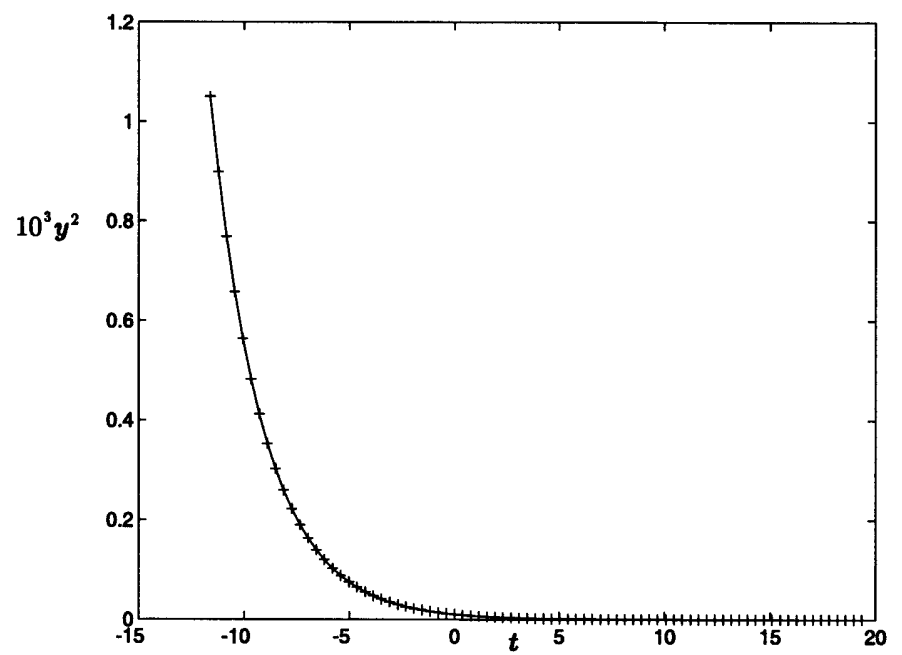

Fig. 6. Noise-free data: $(+) y\left(t_{i}\right)=10^{-5} \exp \left(-0.4 t_{i}\right)+10^{-9} \exp (-$ $\left.0.8 t_{i}\right) ; i=1, \ldots, 81$, and VH model output for $\hat{\boldsymbol{P}}$ obtained by minimizing a least-square criterion with the Gauss-Newton algorithm (continuous line); although the fit looks perfect, $\hat{\boldsymbol{P}}$ is widely off the mark.

of the two vectors $\boldsymbol{p}^{\mathrm{a}}=\boldsymbol{p}^{*}$ and $\boldsymbol{p}^{\mathrm{b}}$, which confirms numerically the result of the structural identifiability study. A third parasitic component, which could not have been predicted by the identifiability study is also present. It is made of undetermined boxes, centered on $\left(p_{1} \in\left[10^{-9} ; 10^{-5}\right] ; \quad p_{2} \in\left[10^{-9} ; 10^{-5}\right] ; \quad p_{3}=0.4 ; \quad p_{4}=0.4\right)$ (see Fig. 5, left). Its volume can be reduced using a smaller $\varepsilon$, but computational time then increases. This area corresponds to a one-exponential model $\left(p_{3}=p_{4}\right)$. This is because the ratio of amplitudes and the large difference between the time constants impose the supremacy of one exponential over the other: the system is said to be ill-conditioned.

It seems worthwhile to note that all the local iterative methods that we tried on this example, including the simplex, Gauss-Newton, Levenberg-Marquardt, conjugate gradient, quasi-Newton, and sequential quadratic programming methods, tend to converge to the parasitic region. The estimated parameters $\hat{p}_{i}$ provided by the Gauss-Newton algorithm are given in Table 3, and the corresponding model output is presented on Fig. 6. Although the value of the cost function is very small $\left(1.00619 \times 10^{-6}\right)$ and the model output seems satisfactory, $\hat{\boldsymbol{p}}$ does not correspond to the global minimizer $\boldsymbol{p}^{*}$, for which the cost would be zero in this noise-free case. As can be checked on Table 3, $\hat{\boldsymbol{p}}$ is actually quite far from $\boldsymbol{p}^{*}$, whereas $\boldsymbol{p}^{*}$ does belong to the set characterized by set inversion.

Set inversion can also be used to eliminate model structures that are inconsistent with the data given their uncertainty. In the case where this uncertainty is negligible, as in the noise-free example, this allows one to study the distinguishability of model structures in a numerical way. We take the one-exponential model described by Eq. (7), and estimate its parameter vector for the Volmer-Heyrovsky noise-free simulated data. Point estimators will find a solution, as we showed that these data can easily be fitted to a one-exponential model, thus accepting the redox model: numerically, one would thus conclude that the redox model is not distinguishable from the Volmer-Heyrovsky model. On the other hand, provided that $\varepsilon$ is taken small enough, SIVIA allows us to prove numerically that the redox model is inconsistent with the data generated according to the Volmer-Heyrovsky mechanism.

We take now $P=P^{2}=\left(\left[10^{-6} ; 10^{6}\right]\left[10^{-9} ; 10^{3}\right][0 ; 1]\right.$ $[0 ; 1])^{\prime}$, which eliminates one of the two solutions due to a lack of global identifiability and which amounts to assuming that:

$$
\left(\begin{array}{c}
k_{01} \in\left[10^{-6} ; 10^{6}\right] \\
k_{02} \in\left[10^{-6} ; 10^{6}\right] \\
\alpha_{01} \in[0 ; 1] \\
\alpha_{02} \in[0 ; 1]
\end{array}\right)
$$

We add $5 \%$ noise to the data, and set:

$[y]_{i}=\left[\left[0.95 \times\left|y_{i}\right| ; 1.05 \times\left|y_{i}\right|\right]\right.$ for $i=1, \ldots, N$.

The estimation of $p_{4}$ becomes very inaccurate (Fig. 5, right). Note that a least-square method would give even worse results than in the noise-free case. The failure should not be blamed upon the estimation method, which is only the bearer of bad news, but upon the data. For such ill-conditioned problems, no estimation method, sophisticated as it may be, can improve the situation. The solution is rather to design the experiment in such a way as to make the data more informative, for instance by reducing $c_{\mathrm{A} \text {,init }}$ or by resorting to impedance measurements. SIVIA can thus help one to conceive better experiments to be performed in order to improve the accuracy of the estimates.

\section{Conclusions}

While the numerical values of the parameters computed by local iterative optimization of a criterion can usually not even be guaranteed to correspond to a global optimizer of this criterion, using set inversion via interval analysis allows one to characterize the set of all 
acceptable parameter vectors in a guaranteed way. Guaranteed means here that no acceptable value of the parameter vector located in the search domain has been eliminated. The accuracy of the enclosure of the solution set $S$ is tuned by the parameter $\varepsilon$. For any given positive $\varepsilon, S$ is guaranteed to be enclosed in the computed outer approximation $S^{+}$. The smaller $\varepsilon$ is, the more accurate the enclosure, at the cost of more complex computations; a compromise must therefore be struck.

Various models can be considered, for which classical estimators may fail, because of structural defects of the model, such as unidentifiability of a given model or indistinguishability of a set of models, or because of numerical problems, such as ill-conditioning. The redox and Volmer-Heyrovsky examples considered in this paper are cases in point. Moreover, as the solutions provided are guaranteed, SIVIA allows one to invalidate hypotheses or models, by proving that the set of feasible parameters is empty.

The algorithm presented here has two major limitations. First, its complexity is exponential in the number of parameters, which restricts its use to low-dimensional problems. For the sake of simplicity of exposition, the version of SIVIA presented here is far from optimal from the viewpoint of computational time, and significant improvements can be expected in the near future. The second limitation is that efficient functions are needed, which are available only when an explicit solution for the equations defining the model can be found. The computation of inclusion functions for differential equations is an active domain of research in interval analysis.

The importance of a realistic specification of the template that defines the acceptable model outputs must be stressed. The approach used here for this specification was to base it on the technical data of the measuring apparatus, but other options could equally be considered.

The steady-state data used in this paper often turn out not to be informative enough, and more accurate results can be expected from impedance data. This requires some extensions of the algorithm, presently under study.

\section{Acknowledgements}

The authors wish to thank B. Le Gorrec, C. Montella and J.-P. Diard for providing experimental data and for fruitful discussions.

\section{References}

[1] B.A. Boukamp, Solid State Ionics 20 (1986) 31.

[2] J.R. Dygas, M.W. Breiter, Electrochim. Acta 44 (1999) 4163.

[3] M.E. Orazem, P. Agarwal, J. Electrochem. Soc. 143 (1996) 948.

[4] T.J. VanderNoot, I. Abrahams, J. Electroanal. Chem. 448 (1998) 17.

[5] G. Salié, J. Electroanal. Chem. 447 (1998) 211.

[6] F. Berthier, J.-P. Diard, C. Montella, L. Pronzato, E. Walter, J. Chim. Phys. 90 (1993) 2069.

[7] F. Berthier, J.-P. Diard, C. Montella, L. Pronzato, E. Walter, J. Electroanal. Chem. 399 (1995) 1.

[8] A.A. Zhiglavsky, Theory of Global Random Search, Kluwer, Dordrecht, 1991.

[9] W.H. Press, S.A. Teukolsky, Numerical Recipes in C: The Art of Scientific Computing, second ed, Cambridge University Press, Cambridge, 1995.

[10] L. Darrel Whitley (Eds.), Foundations of Genetic Algorithms 2, Morgan Kaufmann Publishers, San Mateo, 1993.

[11] E. Walter, L. Pronzato, Identification of Parametric Models from Experimental Data, Springer-Verlag, London, 1997.

[12] A.A. Istratov, O.F. Vyvenko, Am. Inst.Phys. 70 (1999) 1233.

[13] R. Horst, H. Tuy, Global Optimization, Springer-Verlag, Berlin, 1990.

[14] E.R. Hansen, J. Optim. Theor. Appl. 29 (1979) 331.

[15] M. Kieffer, E. Walter, in: A.C. Atkinson, L. Pronzato, H.P. Wynn (Eds.), MODA 5-Advances in Model-Oriented Data Analysis and Experiment Design, Physica Verlag, Heidelberg, 1998, pp. 115-125.

[16] L. Jaulin, E. Walter, Automatica 29 (1993) 1053.

[17] L. Jaulin, E. Walter, Math. Comput. Simulation 35 (1993) 1923.

[18] J.-P. Diard, B. Le Gorrec, C. Montella, Cinétique Electrochimique, Hermann Collection Méthodes, 1996.

[19] F. Berthier, J.-P. Diard, L. Pronzato, E. Walter, Automatica 32 (1996) 973.

[20] M. Milanese, J. Norton, H. Piet-Lahanier, E. Walter, Bounding Approaches to System Identification, New York, Plenum Press, 1996.

[21] E. Walter (Eds.), Special issue on parameter identification with error bounds, Mathematics and Computers in Simulation, 321990 pp. 447.

[22] J.P. Norton (Eds.), Special issue on bounded-error estimation: Int. J. of Adaptive Control and Signal Processing, Issue 1, 81994 pp. 1.

[23] J.P. Norton (Eds.), Special issue on bounded-error estimation: Int. J. of Adaptive Control and Signal Processing, Issue 2, 91995 pp. 1.

[24] Interface Electrochimique, Technical Report, Schlumberger Instruments, 12861985.

[25] E.R. Hansen, Global Optimization using Interval Analysis, Dekker, New York, 1992.

[26] R.E. Moore, Interval Analysis, Prentice-Hall, Englewood Cliffs, New Jersey, 1966.

[27] http://www.mscs.mu.edu/126 globsol/JavaIntervalCalc/I_C.html.

[28] http://www.cs.utep.edu/interval-comp/main.html.

[29] http://www.ti3.tu-harburg.de/126zemke/b4m.

[30] L. Jaulin, Automatica, in press.

[31] F. Benhamou, F. Goualard, L. Granvilliers, J.F. Puget, International Conference on Logic Programming, 1999.

[32] in: J.-P. Diard, B. Le Gorrec, C. Montella, in: C. Gabrielli (Ed.), Proceedings of the Electrochemical Impedance Forum, (1991) p. 145. 